\title{
Research on the Application of AHP and Fuzzy Evaluation in Quota Design
}

\author{
Jing Luo \\ Department of economics and management, North China Electric Power University, Baoding, \\ Hebei, China \\ lj521013@qq.com
}

Keywords: Quota design, FAST graphic method, Principal component analysis, Fuzzy evaluation.

\begin{abstract}
In view of functional analysis is the key work of quota design, we establish the AHP model based on FAST diagram analysis method and principal component analysis. It can design the system function so as to provide a scientific basis for investment quota allocation. On this basis, we establish the fuzzy function evaluation model, this model can comprehensive evaluation of overall function of the design. On one hand, it demonstrate the feasibility of the AHP function analysis model. On the other hand, it provides a simple method for the comprehensive evaluation of the overall function of scientific design.
\end{abstract}

\section{Introduction}

Quota design is a method of effective control of investment. The key problem in quota design is the allocation of investment. Pengfei Li pointed out that as a combination of technology and economy, value engineering caters to the goal of reducing costs and improving performance. It can play a big role in limit design. Haoran Huang, Shouhua Yu have enumerated the concrete application of the analytic hierarchy process in the object selection, the function evaluation and the plan choice in the value engineering activity. Junjie Zhou pointed out that the AHP for the biggest role is the cost control of the whole process, it has changed the traditional way of cost control, the thinking process of cost management into quantitative analysis, and can be carried out by checking the consistency of expert opinion inconsistent treatment. Zhen Ye conducted the research on the fuzzy comprehensive evaluation method based on AHP, using the AHP to establish the evaluation index system, and calculate the weights based on fuzzy comprehensive evaluation method to establish evaluation model, which makes the evaluation more objective.

Introducing value theory into quota design, there are still some problems such as strong subjectivity of function analysis and weak scientific basis of investment allocation. For this reason, we establish a AHP model based on FAST graphical method, systematically analyzes and designs the functions of the design scheme, thus providing a scientific basis for the allocation of investment quotas. On this basis, we establish the fuzzy mathematics function evaluation model, it can evaluate the overall function of the design scheme comprehensively.

\section{The Function Evaluation Model of Quota Design}

\subsection{AHP Model based on FAST Graphical Method}

Functional analysis is the core of value engineering. Functional analysis and arrangement are prerequisites for a more accurate evaluation of functions, so the preciseness and hierarchy of functional arrangement are very important. In order to improve the effectiveness of functional finishing, we introduced FAST graphical method for the selection and determination of the elements of the index layer in constructing the analytic hierarchy model. It can make the AHP model more systematic. 
(1) Determine the VE object

The VE object is determined based on combining the AHP method and the FAST graphical method. We make the VE research object as a whole, and then set the overall objective for the VE "to limit" function. We learn from the corresponding knowledge of demand as a function of the first level of function.

(2) Establish a hierarchical structure based on FSAT graphical method

We draw a hierarchical structure diagram based on combining the FAST graphic method and AHP. Figure 1 shows the hierarchical structure diagram.

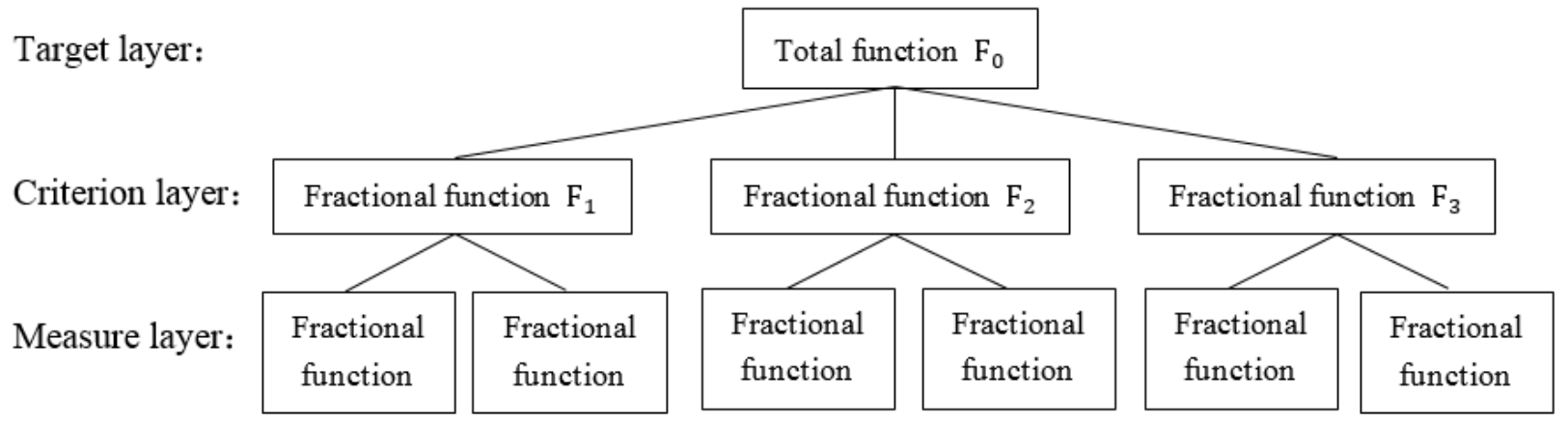

(3) Model solving

Figure 1 The hierarchical structure diagram

On the basis of the above work, we construct the judgment matrix do and the hierarchical single sorting and consistency checking. We can obtain the weight values of each function can through the hierarchical total sorting.

\subsection{Scheme Evaluation Model}

On the basis of AHP model, we establish a scheme evaluation model based on fuzzy mathematics and get a comprehensive evaluation of the design scheme. It include the following steps:

(1) Determine the factor set and the comment set. The factor set is U, and the set consisting of various decisions is the comment set, which is denoted as $\mathrm{V}$.

$$
\boldsymbol{U}=\left\{u_{1}, \ldots, u_{n}\right\}, \boldsymbol{V}=\left\{v_{1}, \ldots, v_{n}\right\}
$$

(2) Determine the weight of each factor. It is a fuzzy vector on $U$.

$$
\boldsymbol{A}=\left\{a_{1}, \ldots, a_{n}\right\}
$$

(3) Determine the fuzzy comprehensive judgment matrix. The evaluation of the target $u_{i}$ is recorded as

$$
R_{i}=\left[r_{i 1}, \ldots, r_{i m}\right]
$$

The fuzzy comprehensive judgment matrix of each index is

$$
R=\left[\begin{array}{cccc}
r_{11} & r_{12} & \cdots & r_{1 m} \\
r_{21} & r_{22} & \cdots & r_{2 m} \\
\vdots & \vdots & \ddots & \vdots \\
r_{n 1} & r_{n 2} & \cdots & r_{n m}
\end{array}\right]
$$

(4) Fuzzy comprehensive evaluation, if there is a fuzzy relation from $\mathrm{U}$ to $\mathrm{V}$

$$
T_{R}: \mathrm{F}(\mathrm{U}) \rightarrow \mathrm{F}(\mathrm{V})
$$

Then, we can obtain a fuzzy transformation by using $\mathrm{R}$.

Comprehensive evaluation results can be obtained. $\mathrm{B}=\mathrm{AR}$. Comprehensive evaluation can be regarded as a fuzzy vector $\mathrm{V}$, denoted by $\mathrm{B}=\left[\mathrm{b}_{1}, \cdots, \mathrm{b}_{\mathrm{m}}\right]$.

\section{Example Analysis}

\subsection{Raw Data}

In the design of a residential project as the research object, collect information on all aspects of the project, the establishment of AHP model FAST graphic method based on function analysis and calculation of the function weight coefficients are proposed using the project model, a solid foundation for the evaluation of a function. 


\subsection{Function Analysis}

\subsubsection{Hierarchical Structure based on FAST Graphical Method}

Combined with FAST graphic method, we determine the target layer is the proposed project. According to the basic principle of FAST diagram method, we analyze each function of the proposed project, which is defined as the criterion layer. In this example, the criterion layer contains 5 sub functions. Table 1 shows the selection of the sub functions. Because of the influence of the design function of measure layer, the index is a complex problem, so the measures involved may be too many. Therefore, we use principal component analysis to optimize the measure layer index, so that the calculation and analysis is more concise and scientific. Table 2 shows the AHP hierarchy based on the FAST graphical method.

Table 1. Selection of the sub functions

\begin{tabular}{|c|c|}
\hline $\begin{array}{l}\text { Index of } \\
\text { sources }\end{array}$ & Technical standards for performance evaluation of residential buildings \\
\hline Index name & $\begin{array}{c}\text { Performance } \mathrm{F}_{1} \text {, Environmental performance } \mathrm{F}_{2} \text {, Economic performance } \mathrm{F}_{3} \text {, Safety } \\
\text { performance } \mathrm{F}_{4} \text {, Durability } \mathrm{F}_{5}\end{array}$ \\
\hline \multicolumn{2}{|c|}{ Table 2. AHP hierarchy based on FAST graphical method. } \\
\hline The criterion layer & The measure layer \\
\hline Performance $\mathrm{F}_{1}$ & $\begin{array}{c}\text { Flat dwelling flat } F_{11} \text {; Building decoration } F_{12} \text {; Sound insulation performance } F_{13} \text {; } \\
\text { Equipment implementation } F_{14}\end{array}$ \\
\hline $\begin{array}{l}\text { Environmental } \\
\text { performance } \mathrm{F}_{2}\end{array}$ & $\begin{array}{l}\text { Land use and planning } F_{21} \text {; Greening } F_{22} ; \text { Drainage systems } F_{23} \text {; Outdoor noise } \\
\text { pollution } F_{24}\end{array}$ \\
\hline Economic performance $\mathrm{F}_{3}$ & Energy saving $F_{31} ;$ Water saving $F_{32} ;$ Land saving $F_{33} ;$ Material saving $F_{34}$ \\
\hline Safety performance $\mathrm{F}_{4}$ & Structural safety $F_{41}$; Electrical equipment safety $F_{42}$; Daily safety Fire safety $F_{43}$ \\
\hline Durability $\mathrm{F}_{5}$ & $\begin{array}{c}\text { Structure and property } F_{51} \text {; Waterproof and damp proof pipeline } F_{52} ; \text { Equipment } \\
\text { performance } F_{53}\end{array}$ \\
\hline
\end{tabular}

\subsubsection{Functional Analysis}

We can obtain the total weight of the measure layer relative to the target layer by the hierarchical total ordering. Table 3 shows the overall ranking results of the design plan of the district construction project. It can be seen that the weight coefficient and the order of the target layer for the target layer, that is, the weight coefficient and order of each function that affect the allocation of investment. We should allocate the amount of investment according to the proportion of weighting factors. That is, for the function value coefficient, the overall sorting function, focusing on the design. And then we can make the design limit of the control and control are playing a better control role.

Table 3. The overall ranking results

\begin{tabular}{|c|c|c|c|c|c|c|}
\hline Target layer, & Criterion layer & B to A & Measure layer & C to $\mathrm{B}$ & $\mathrm{C}$ to $\mathrm{A}$ & C to A \\
\hline \multirow{20}{*}{ A } & \multirow{5}{*}{$\mathrm{B}_{1}$} & \multirow{5}{*}{0.276} & $\mathrm{c}_{11}$ & 0.263 & 0.0725 & 4 \\
\hline & & & $\mathrm{c}_{12}$ & 0.393 & 0.1084 & 3 \\
\hline & & & $\mathrm{c}_{13}$ & 0.129 & 0.0356 & 9 \\
\hline & & & $\mathrm{c}_{14}$ & 0.092 & 0.0253 & 10 \\
\hline & & & $\mathrm{c}_{15}$ & 0.072 & 0.0198 & 12 \\
\hline & \multirow{4}{*}{$\mathrm{B}_{2}$} & \multirow{4}{*}{0.056} & $c_{21}$ & 0.130 & 0.0072 & 17 \\
\hline & & & $\mathrm{c}_{22}$ & 0.098 & 0.0055 & 19 \\
\hline & & & $\mathrm{c}_{23}$ & 0.062 & 0.0035 & 20 \\
\hline & & & $c_{24}$ & 0.205 & 0.0115 & 16 \\
\hline & \multirow{4}{*}{$\mathrm{B}_{3}$} & \multirow{4}{*}{0.132} & $c_{31}$ & 0.116 & 0.0153 & 15 \\
\hline & & & $c_{32}$ & 0.138 & 0.0182 & 14 \\
\hline & & & $c_{33}$ & 0.432 & 0.0570 & 5 \\
\hline & & & $c_{34}$ & 0.332 & 0.0438 & 7 \\
\hline & \multirow{4}{*}{$\mathrm{B}_{4}$} & \multirow{4}{*}{0.448} & $\mathrm{c}_{41}$ & 0.436 & 0.1953 & 1 \\
\hline & & & $\mathrm{c}_{42}$ & 0.276 & 0.1236 & 2 \\
\hline & & & $\mathrm{c}_{43}$ & 0.125 & 0.0560 & 6 \\
\hline & & & $\mathrm{c}_{44}$ & 0.043 & 0.0193 & 13 \\
\hline & \multirow{3}{*}{$\mathrm{B}_{5}$} & \multirow{3}{*}{0.088} & $c_{51}$ & 0.469 & 0.0412 & 8 \\
\hline & & & $c_{52}$ & 0.226 & 0.0199 & 11 \\
\hline & & & $c_{53}$ & 0.063 & 0.0055 & 18 \\
\hline
\end{tabular}




\subsection{Function Evaluation}

We make a comprehensive evaluation scheme of a certain area of the proposed project. The evaluation grade is 5 and $\mathrm{u}_{\mathrm{i}}$ correspond $\mathrm{c}_{11}, \cdots, \mathrm{c}_{53}$, that is,

$$
\mathrm{U}=\left\{\mathrm{u}_{1}, \mathrm{u}_{2}, \cdots \mathrm{u}_{20}\right\}
$$

$$
\mathrm{V}=\left\{\mathrm{v}_{1}, \mathrm{v}_{2}, \cdots \mathrm{v}_{5}\right\}=\{\text { poor, average, medium, good, excellent }\}
$$

In order to improve the accuracy of the model, the model using the AHP weight value derived. Table 3 shows the weight value, namely $\mathrm{W}=\left(\begin{array}{llll}0.276 & 0.056,0.132,0.448,0.088\end{array}\right)^{T}$.

According to the results of experts evaluation, with the score divided by 50 as the membership, we can obtain the single factor evaluation matrix of $B_{1}, B_{2}, B_{3}, B_{4}, B_{5}$. The single factor evaluation matrices $R_{2}, R_{3}, R_{4}, R_{5}$ are similar to $R_{1}$. We evaluate he fuzzy relation matrix of each criterion layer by MATLAB. The fuzzy relation total matrix $\mathrm{Q}$ is obtained by each fuzzy relation matrix

$$
Q=\left[\begin{array}{lllll}
0.0393 & 0.2119 & 0.3593 & 0.2428 & 0.1466 \\
0.0300 & 0.2251 & 0.4050 & 0.2451 & 0.0950 \\
0.0592 & 0.1212 & 0.3038 & 0.3483 & 0.1644 \\
0.0500 & 0.1700 & 0.3150 & 0.3300 & 0.1357 \\
0.0225 & 0.0675 & 0.2775 & 0.4350 & 0.2100
\end{array}\right]
$$

Through the fuzzy transformation of the weight coefficient matrix $\mathrm{W}$ and the evaluation matrix $\mathrm{R}$, we obtain the fuzzy evaluation set $\mathrm{S}$.

$$
\mathrm{S}=\mathrm{w}^{\circ} \mathrm{R}=\left(\mu_{1}, \mu_{2}, \cdots \mu_{\mathrm{m}}\right) \circ\left[\begin{array}{cccc}
r_{11} & r_{12} & \cdots & r_{1 n} \\
r_{21} & r_{22} & \cdots & r_{2 n} \\
\cdots & \cdots & \vdots & \cdots \\
r_{m 1} & r_{m 2} & \cdots & r_{m n}
\end{array}\right]=\left(s_{1}, \mathrm{~s}, \cdots s_{n}\right)
$$

Among them, the "degree" is the fuzzy synthesis operator, and the fuzzy synthesis operator include $M(\Lambda, \vee), M(\bullet, \vee), M(\Lambda, \oplus), M(\bullet, \oplus)$ we use the optimal fuzzy comprehensive operator $\mathrm{M}(\Lambda, \oplus)$ to calculate.

$$
\mathrm{S}=\mathrm{W}^{\circ} \mathrm{R}=(0.04826,0.1657,0.1393,0.3095,0.3356)
$$

According to the principle of maximum degree of membership, the function evaluation of design scheme for residential construction project "excellent". This proves the superiority of AHP model for function analysis and weight allocation, and also provides a concise method for the comprehensive evaluation of the overall function of the design scheme.

\section{Conclusion}

(1) We establish the AHP model based on FAST graphical method and PCA. The FAST graphical method can make the function analysis of the design scheme more systematic and accurate, and PCA plays an important role in the optimization of measure level indexes. The model can be used to analyze the function of the design plan systematically, and provide a scientific basis for the allocation of investment quota.

(2) We establish the fuzzy mathematics function evaluation model of AHP. It can evaluate the overall functional design on the basis of the AHP model, which not only demonstrate the superiority of AHP function analysis model on the one hand, but also provide method of concise and scientific evaluation for overall function design.

\section{References}

[1]. Shoukui Si, Xijing Sun. Modeling and application of mathematics. National Defense Industry Press, 2015.

[2]. Zhen Ye. Research and application of fuzzy comprehensive evaluation method based on AHP [D]. South China University of Technology, 2010.

[3]. Xue Deng, Gang Li, Wei Zeng, Junyang Chen, Junfeng Zhao. Analysis and application of analytic hierarchy process weights. [J]. practice and understanding of mathematics, 2012, 07:93100. 
[4]. Jiang Wei. Study on Application of limit design method in cost control [D]. South China University of Technology, 2014.

[5]. Pengfei Li. Application of value engineering in quota design of construction projects [D]. University of Chinese Academy of Sciences (Engineering Management and Information Technology Institute), 2014.

[6]. Junjie Zhou. Study on the whole process cost control of rail transit engineering based on AHP analytic hierarchy process [D]. Shanghai Jiao Tong University, 2014. 